\title{
Trial Disease Minimum Time Allowed in Planned Assessment
}

National Cancer Institute

\section{Source}

National Cancer Institute. Trial Disease Minimum Time Allowed in Planned Assessment. NCl Thesaurus. Code C117704.

The lower limit in time of a planned period of time between planned trial disease assessments. 